\title{
IMMERSION OF MANIFOLDS WITH UNBOUNDED IMAGE AND A MODIFIED MAXIMUM PRINCIPLE OF YAU
}

\author{
ALBERT BORBÉLY
}

(Received 8 January 2008)

\begin{abstract}
Let $N$ be a complete Riemannian manifold isometrically immersed into a Hadamard manifold $M$. We show that the immersion cannot be bounded if the mean curvature of the immersed manifold is small compared with the curvature of $M$ and the Laplacian of the distance function on $N$ grows at most linearly. The latter condition is satisfied if the Ricci curvature of $N$ does not approach $-\infty$ too fast. The main tool in the proof is a modification of Yau's maximum principle.
\end{abstract}

2000 Mathematics subject classification: 53C42.

Keywords and phrases: immersion, mean curvature, maximum principle.

\section{Introduction}

Let $N$ be a complete Riemannian manifold isometrically immersed into a Hadamard manifold $M$; in other words, $M$ is a complete, simply connected manifold with nonpositive curvature. We are looking for conditions that force the immersion to be unbounded. We impose two conditions. First, we require the immersion to be close to a minimal immersion; that is, the mean curvature of the immersion must be small. Second, we require that the Laplacian of the distance function on $N$ grows at most linearly.

It is perhaps unusual to impose an analytical condition on the immersed manifold, rather than a geometrical one (involving bounds on the curvatures); but our reasons are two-fold.

First of all, there is a strong connection between the Laplacian of the distance function and the Ricci curvature. For instance, a lower bound on the Ricci curvature would imply, via standard comparison arguments, an upper bound on the Laplacian of the distance function for distances larger than one. By the same token, a decay condition on the Ricci curvature (which says that the Ricci curvature cannot approach $-\infty$ faster than a certain rate) yields a growth condition on the Laplacian of the distance function.

(c) 2008 Australian Mathematical Society 0004-9727/08 \$A2.00+ 0.00 
The second reason is that this analytical condition on the immersed manifold is the property that will play an especially important role in the proofs.

It is also clear that we must impose some condition on the immersed manifold, either in the form of a curvature condition or in terms of the Laplacian of the distance function, because there are examples [1] of complete immersed minimal surfaces into $\mathbb{R}^{3}$ that are bounded.

Before we proceed to the theorem, it will be convenient to formulate in a precise manner the analytical condition we need.

Condition $(\Delta)$. We say that the Riemannian manifold $N^{n}$ satisfies Condition $(\Delta)$ if there exist a point $p \in N$ and a positive number $C>0$ such that $\Delta r(x)<C r(x)$ for all $x \in N$, where $r$, the distance function from $p$ on $N$, is smooth with $r(x)>1$.

Our result can now be formulated as follows.

THEOREM. Let $N^{n}$ be a complete Riemannian manifold satisfying Condition $(\Delta)$. Let $M^{m}$ be a complete simply connected manifold with sectional curvatures $K \leq-a^{2}$, where $a \geq 0$ and $m>n$. Let $f: N^{n} \rightarrow M^{m}$ be an isometric immersion such that the norm of the mean curvature vector of $f$ satisfies $\left\|H_{f}\right\| \leq a(n-1) / n$. Then $f\left(N^{n}\right)$ is not bounded in $M^{m}$.

The important tool in the proof of this theorem is the following modified version of Yau's maximum principle [3, Theorem 1].

LEMMA. Let $N^{n}$ be a complete Riemannian manifold satisfying Condition $(\Delta)$ and let $g: N^{n} \rightarrow \mathbb{R}$ be a smooth function that is bounded from above. Then, for every $\varepsilon>0$, there is a point $x_{\varepsilon} \in N^{n}$ such that $\left|g\left(x_{\varepsilon}\right)-\sup g\right|<\varepsilon,\left\|\nabla g\left(x_{\varepsilon}\right)\right\|<\varepsilon$ and $\Delta g\left(x_{\varepsilon}\right)<\varepsilon$.

The point $x_{\varepsilon}$ in the lemma plays the role of an 'approximate maximum'.

Let us remark that the condition $\Delta r=O(r)$ is quite optimal. For manifolds with $\Delta r>r^{1+\varepsilon}$ for large $r$, it is easy to construct a bounded function for which the lemma does not apply. The details can be found at the end of this paper.

\section{Proofs}

Proof of THE THEOREM. Let us assume, on the contrary, that $f(N)$ is bounded in $M$.

Let $O \in M-N$ be any fixed point, and denote by $r: M \rightarrow \mathbb{R}^{+}$the distance function from $O$ on $M$. Let $r_{\mid N}: N \rightarrow \mathbb{R}^{+}$be the restriction of $r$ to $N$, that is, $r_{\mid N}(x)=r(f(x))$. Our goal is to compute $\Delta r_{\mid N}$.

To simplify notation and to make the calculations easier to follow, we shall identify the manifold $N$ with its image $f(N)$. This has the advantage that we do not need to push forward (and pull back) tangent vectors of $N$ to tangent vectors of $M$ via $d f: T N \rightarrow T M$. Since $f: N \rightarrow M$ is an isometric immersion, this idenitification is justified.

Let $\nabla$ denote the connection on $M, \widetilde{\nabla}$ the connection on $N$ and $\langle.,$.$\rangle the Riemannian$ metric on $M$. 
Let $q \in N$; near $q$, we can decompose $\nabla r$ into tangential and normal components of $T N$,

$$
\nabla r=(\nabla r)^{\mathrm{T}}+(\nabla r)^{\mathrm{N}}
$$

and we have the relation

$$
\nabla r_{\mid N}=(\nabla r)^{\mathrm{T}}
$$

For tangent vectors $X, Y \in T_{q} N$,

$$
\begin{aligned}
\operatorname{Hess}\left(r_{\mid N}\right)(X, Y) & =\left\langle\widetilde{\nabla}_{X} \nabla\left(r_{\mid N}\right), Y\right\rangle=\left\langle\nabla_{X}\left(\nabla r-(\nabla r)^{\mathrm{N}}\right), Y\right\rangle \\
& =\operatorname{Hess} r(X, Y)-\left\langle\nabla_{X}(\nabla r)^{\mathrm{N}}, Y\right\rangle .
\end{aligned}
$$

Since the normal component $(\nabla r)^{\mathrm{N}}$ of the gradient is orthogonal to $N$, it follows that

$$
\left\langle\nabla_{X}(\nabla r)^{\mathrm{N}}, Y\right\rangle=-\left\langle(\nabla r)^{\mathrm{N}}, \nabla_{X} Y\right\rangle=\left\langle(\nabla r)^{\mathrm{N}}, A(X, Y)\right\rangle,
$$

where $A(X, Y)=-\left(\nabla_{X} Y\right)^{\mathrm{N}}$ denotes the vector-valued second fundamental form of $N$. Combining this with the equality above, we obtain

$$
\operatorname{Hess}\left(r_{\mid N}\right)(X, Y)=\operatorname{Hess} r(X, Y)-\left\langle(\nabla r)^{\mathrm{N}}, A(X, Y)\right\rangle \text {. }
$$

Let $\lambda$ be a nonzero eigenvalue of $r$ at $x \in M$. Since $M$ is a Hadamard manifold with sectional curvature $K \leq-a^{2}$, a standard comparison argument [2, Ch. 6] gives us that $\lambda \geq a \operatorname{coth}(\operatorname{ar}(x))$ if $a>0$, and $\lambda \geq 1 / r(x)$ if $a=0$. Since we assumed that $f(N)$ is bounded in $M$, there is a $\delta>0$, depending only on the bound of $f(N)$, such that for all points of $N, \lambda>a+\delta$. This implies that for any unit vector $X \in T_{q} N$ orthogonal to $\nabla r^{\mathrm{T}}$, we have Hess $r(X, X)>a+\delta$.

Taking the trace in (1) with respect to $T_{q} N$, we get

$$
\Delta\left(r_{\mid N}\right)>(n-1)(a+\delta)-\left\langle n H_{f}(q), \nabla r^{\mathrm{N}}\right\rangle \geq(n-1)(a+\delta)-n\left\|H_{f}(q)\right\|,
$$

where $H_{f}=(1 / n) \operatorname{Trace}(A)$ denotes the mean curvature vector of $N$.

Since we assumed that $\left\|H_{f}\right\| \leq a(n-1) / n$, it follows that

$$
\Delta\left(r_{\mid N}\right)>(n-1) \delta,
$$

for every point of $N$. But this clearly contradicts the lemma, as $r_{\mid N}$ is a bounded smooth function on $N$. The proof of the theorem is thus complete.

Proof of The Lemma. Set $L=\sup g$. We can assume that $g<L$ at every point of $N$; otherwise, $g$ must assume its maximum at some point, and that point would trivially satisfy the conditions of the lemma for all $\varepsilon>0$.

Let $p \in N$ be the point and $C>0$ the positive constant in Condition ( $\Delta$ ); also let $r$ be the distance function from $p$ on $N$.

For any $\varepsilon<L-\sup \{g(x) \mid r(x) \leq 1\}$, define the function $h_{\lambda}: N \rightarrow \mathbb{R}$ by

$$
h_{\lambda}(x)=\lambda r(x)+L-\varepsilon .
$$


Clearly,

$$
h_{\lambda}(x)>g(x) \quad \text { if } r(x) \leq 1 \text { and } \lambda \geq 0 .
$$

Therefore, if $\lambda>\varepsilon$, then $h_{\lambda}(x)>g(x)$ for all $x \in N$.

Define $\lambda_{0}$ as

$$
\lambda_{0}=\inf \left\{\lambda \mid h_{\lambda}(x)>g(x) \text { for all } x \in N\right\} .
$$

Since sup $g=L$, it is easy to see that $\lambda_{0}>0$ and $h_{\lambda_{0}}(x) \geq g(x)$ for all $x \in N$.

Next, we claim that there is a point $x_{\varepsilon} \in N$ such that $h_{\lambda_{0}}\left(x_{\varepsilon}\right)=g\left(x_{\varepsilon}\right)$. Let us assume this is not true, that is, $h_{\lambda_{0}}(x)>g(x)$ for all $x \in N$. Set $r_{\varepsilon}=2 \varepsilon / \lambda_{0}$ and denote by $B_{r_{\varepsilon}}=\left\{x \in N \mid r(x) \leq r_{\varepsilon}\right\}$ the closed ball of radius $r_{\varepsilon}$. From the definition of $h_{\lambda}$ it follows that $h_{\lambda_{0}}(x) \geq L+\varepsilon$ for all $x \notin B_{r_{\varepsilon}}$. Using the fact that $B_{r_{\varepsilon}}$ is compact and our assumption that $h_{\lambda_{0}}(x)>g(x)$ for all $x \in N$, one concludes that

$$
\inf _{x \in B_{r_{\varepsilon}}}\left(h_{\lambda_{0}}(x)-g(x)\right)>0 .
$$

Since $g<L$ on $N$,

$$
\inf _{x \in N}\left(h_{\lambda_{0}}(x)-g(x)\right)=\eta>0 .
$$

Now let $0<\delta<\min \{\eta, \varepsilon\}$ be any number and set $\lambda^{\prime}=\lambda_{0}-\delta /\left(2 r_{\varepsilon}\right)$. We shall show that $h_{\lambda^{\prime}}(x)>g(x)$ for all $x \in N$, which would contradict the definition of $\lambda_{0}$.

If $r(x) \leq r_{\varepsilon}$, then

$$
\begin{aligned}
h_{\lambda^{\prime}}(x) & =\left(\lambda_{0}-\frac{\delta}{2 r_{\varepsilon}}\right) r(x)+L-\varepsilon \\
& =h_{\lambda_{0}}(x)-\frac{\delta r(x)}{2 r_{\varepsilon}} \geq g(x)+\eta-\delta \frac{r(x)}{2 r_{\varepsilon}}>g(x) .
\end{aligned}
$$

On the other hand, taking into consideration that $\lambda_{0}-\delta /\left(2 r_{\varepsilon}\right)>0$ because $\lambda_{0} r_{\varepsilon}=2 \varepsilon>\delta$, we deduce, for $r(x)>r_{\varepsilon}$, that

$$
\begin{aligned}
h_{\lambda^{\prime}}(x) & =\left(\lambda_{0}-\frac{\delta}{2 r_{\varepsilon}}\right) r(x)+L-\varepsilon>\left(\lambda_{0}-\frac{\delta}{2 r_{\varepsilon}}\right) r_{\varepsilon}+L-\varepsilon \\
& =L+\varepsilon-\frac{\delta}{2}>L>g(x) .
\end{aligned}
$$

As the assumption has led to a contradiction, we conclude that there is a point $x_{\varepsilon} \in N$ such that $h_{\lambda_{0}}\left(x_{\varepsilon}\right)=g\left(x_{\varepsilon}\right)$. Moreover, from (2) it follows that $r\left(x_{\varepsilon}\right) \geq 1$.

Finally, we have to show that $h_{\lambda_{0}}$ is smooth at $x_{\varepsilon}$. Since $h_{\lambda}(x)=\lambda r(x)+L-\varepsilon$, it is enough to show that $r$ is smooth at $x_{\varepsilon}$. If not, then $x_{\varepsilon}$ must be on the cut locus of $p$, in which case we have two possibilities: either there are two distinct minimizing geodesic segments $\gamma_{1}, \gamma_{2}:\left[0, t_{0}\right] \rightarrow N$ joining $p$ to $x_{\varepsilon}$, or there is a geodesic segment $\gamma:\left[0, t_{0}\right] \rightarrow N$ from $p$ to $x_{\varepsilon}$ along which $x_{\varepsilon}$ is conjugate to $p$.

Let us start with the first case. Let $w=\gamma_{1}^{\prime}\left(t_{0}\right)$ and $v=\gamma_{2}^{\prime}\left(t_{0}\right)$. Since $\gamma_{1}$ and $\gamma_{2}$ are distinct segments, we have $w \neq v$. The functions $t \rightarrow r\left(\gamma_{i}(t)\right)$ are differentiable on $\left(0, t_{0}\right)$ (for $\left.i=1,2\right)$ and they have a left-derivative at $t_{0}$. 
From $h_{\lambda_{0}} \geq g$ and $h_{\lambda_{0}}\left(x_{\varepsilon}\right)=g\left(x_{\varepsilon}\right)$ it follows that

$$
\liminf _{s \rightarrow 0} \frac{h_{\lambda_{0}}\left(\gamma_{2}\left(t_{0}+s\right)\right)-h_{\lambda_{0}}\left(\gamma_{2}\left(t_{0}\right)\right)}{s} \geq D_{v} g\left(x_{\varepsilon}\right),
$$

where $D_{v} g\left(x_{\varepsilon}\right)$ denotes the directional derivative of $g$ at the point $x_{\varepsilon}$ in the direction of $v$. Moreover, since $g$ is smooth and $h_{\lambda_{0}}$ has a directional derivative at $x_{\varepsilon}$ in the direction of $-v$, we also have

$$
-\lambda_{0}=D_{-v} h_{\lambda_{0}}\left(x_{\varepsilon}\right) \geq D_{-v} g\left(x_{\varepsilon}\right)=-D_{v} g\left(x_{\varepsilon}\right) .
$$

Combining this with the previous inequality, we obtain

$$
\liminf _{s \rightarrow 0} \frac{h_{\lambda_{0}}\left(\gamma_{2}\left(t_{0}+s\right)\right)-h_{\lambda_{0}}\left(\gamma_{2}\left(t_{0}\right)\right)}{s} \geq \lambda_{0} .
$$

Taking into account the special form of $h_{\lambda_{0}}$, we find that

$$
\liminf _{s \rightarrow 0} \frac{r\left(\gamma_{2}\left(t_{0}+s\right)\right)-r\left(\gamma_{2}\left(t_{0}\right)\right)}{s} \geq 1
$$

This will lead to a contradiction.

Since $v \neq w$, there is a $0<c<1$, depending only on the angle of $v$ and $w$, such that

$$
r\left(\gamma_{2}\left(t_{0}+s\right)\right)<t_{0}+c s
$$

for a small enough $s>0$. One can see this by connecting the point $\gamma_{1}\left(t_{0}-s\right)$ to the point $\gamma_{2}\left(t_{0}+s\right)$ by a geodesic segment; as $\gamma_{1}$ and $\gamma_{2}$ are different, there is a $0<c_{1}<1$ such that for a small enough $s>0$ we have $\operatorname{dist}\left(\gamma_{1}\left(t_{0}-s\right), \gamma_{2}\left(t_{0}+s\right)\right)<c_{1} 2 s$, which implies (5). But since $r\left(\gamma_{2}\left(t_{0}\right)\right)=t_{0}$, it is easy to see that (4) and (5) are in direct contradiction.

We now turn our attention to the second case. Since $\gamma$ is distance-minimizing between $p$ and $x_{\varepsilon}$, the distance function $r$ is smooth at $\gamma(t)$ for $0<t<t_{0}$. Set $m(t)=\Delta r(\gamma(t))$; then $m(t)$ is also smooth on the interval $\left(0, t_{0}\right)$ and, since $\gamma\left(t_{0}\right)$ is conjugate to $p=\gamma(0)$ along $\gamma$, it follows that

$$
\lim _{t \rightarrow t_{0}^{-}} m(t)=-\infty
$$

Because $\lambda_{0}>0$ from (3), we conclude that $D_{v} g\left(x_{\varepsilon}\right)>0$, that is, $\nabla g\left(x_{\varepsilon}\right) \neq 0$. This implies that the level surface $F=\left\{x \in N \mid g(x)=g\left(x_{\varepsilon}\right)\right\}$ is a smooth hypersurface near $x_{\varepsilon}$. Denote by $F_{s}$ the surface parallel to $F$ and passing through the point $\gamma\left(t_{0}-s\right)$, for some $s>0$. Again, since $F$ is smooth near $x_{\varepsilon}$, the surface $F_{s}$ will also be smooth near $\gamma\left(t_{0}-s\right)$ for a small enough $s>0$.

It is now clear from (6) that, for some small $s>0$,

$$
m\left(t_{0}-s\right)<\text { trace of the second fundamental form of } F_{s} \text { at } \gamma\left(t_{0}-s\right),
$$


where the second fundamental form of $F_{s}$ at $\gamma\left(t_{0}-s\right)$ is taken in the direction of $\gamma^{\prime}\left(t_{0}-s\right)$.

Taking into account the fact that $m\left(t_{0}-s\right)$ is the trace of the second fundamental form of the geodesic ball $B_{p}\left(t_{0}-s\right)$ around $p$ at the point $\gamma\left(t_{0}-s\right)$ (with respect to the same normal vector $\gamma^{\prime}\left(t_{0}-s\right)$ ), we conclude that there has to be a point $q_{s} \in F_{s}$, sufficiently close to $\gamma\left(t_{0}-s\right)$, that lies inside $B_{p}\left(t_{0}-s\right)$. This means that

$$
r\left(q_{s}\right)<t_{0}-s .
$$

Since $F_{s}$ is parallel to $F$, we have a point $q \in F$ such that $\operatorname{dist}\left(q_{s}, q\right)=s$. Combining this with the above inequality gives

$$
r(q)<t_{0} .
$$

From the above, we obtain

$$
h_{\lambda_{0}}(q)=\lambda_{0} r(q)+L-\varepsilon<\lambda_{0} t_{0}+L-\varepsilon=h_{\lambda_{0}}\left(x_{\varepsilon}\right)=g\left(x_{\varepsilon}\right)=g(q),
$$

which leads to a contradiction since $h_{\lambda_{0}} \geq g$ on $N$.

Once we have established the smoothness of $h_{\lambda_{0}}$ at $x_{\varepsilon}$, the rest of the argument is straightforward. Since

$$
h_{\lambda_{0}}(x)>g(x) \quad \text { and } \quad h_{\lambda_{0}}\left(x_{\varepsilon}\right)=g\left(x_{\varepsilon}\right),
$$

we have

$$
\nabla g\left(x_{\varepsilon}\right)=\nabla h_{\lambda_{0}}\left(x_{\varepsilon}\right)=\lambda_{0} \nabla r\left(x_{\varepsilon}\right) \quad \text { and } \quad \Delta h_{\lambda_{0}}\left(x_{\varepsilon}\right) \geq \Delta g\left(x_{\varepsilon}\right) .
$$

From (2) and the fact that $g\left(x_{\varepsilon}\right)=\lambda_{0} r\left(x_{\varepsilon}\right)+L-\varepsilon<L$, we deduce that

$$
r\left(x_{\varepsilon}\right)>1 \quad \text { and } \quad \lambda_{0}<\frac{\varepsilon}{r\left(x_{\varepsilon}\right)} .
$$

Taking $|\nabla r|=1$ and combining it with the first equality in (7) yields

$$
\left\|\nabla g\left(x_{\varepsilon}\right)\right\|=\lambda_{0}<\varepsilon .
$$

From (7), (8) and Condition $(\Delta)$ we find that

$$
\Delta g\left(x_{\varepsilon}\right) \leq \Delta h_{\lambda_{0}}\left(x_{\varepsilon}\right)=\lambda_{0} \Delta r\left(x_{\varepsilon}\right)<\varepsilon \frac{\Delta r\left(x_{\varepsilon}\right)}{r\left(x_{\varepsilon}\right)}<\varepsilon C .
$$

Since $L-\varepsilon<h_{\lambda_{0}}\left(x_{\varepsilon}\right)=g\left(x_{\varepsilon}\right)<L$, inequalities (9) and (10) show that the point $x_{\varepsilon}$ satisfies the conditions of the lemma (if $C>1$, then just replace $\varepsilon$ by $\varepsilon C$ in the statement of the lemma); therefore the proof is complete. 


\section{An example}

In this section we sketch an example which shows that the condition in the lemma, namely Condition $(\Delta)$, is quite optimal. Let $N$ be a complete unbounded manifold, that is, a manifold where the distance function is unbounded. Let $r$ be the distance function from some point, and assume that $\Delta r(x)>r(x)^{1+s}$ for those points $x \in N$ where $r(x)$ is large enough and $0<s<1$. Then there is a bounded function $h: N \rightarrow \mathbb{R}$ for which the lemma does not apply.

Define $h(x)=1-1 / r(x)^{s}$ for $r(x)>2$ and extend it smoothly over the closed ball $B_{2}=\{x \in N \mid r(x) \leq 2\}$ so that $h \leq 1 / 2$ over $B_{2}$. Clearly, $h$ is a bounded function with $\sup h=1$.

A simple computation shows that, outside the ball $B_{2}$,

$$
\Delta h=-s(1+s) \frac{|\nabla r|^{2}}{r^{2+s}}+s \frac{\Delta r}{r^{1+s}} .
$$

Since $|\nabla r|=1$, there is an $r_{0}>0$ such that if $r(x)>r_{0}$, then $\Delta h>s / 2$. Let us choose $\varepsilon>0$ such that $\varepsilon<1-1 / r_{0}^{s}$ and $\varepsilon<s / 2$. Now, if $x_{\varepsilon} \in N$ is the point in $N$ satisfying the conditions of the lemma, then from the condition $\left|h\left(x_{\varepsilon}\right)-1\right|<\varepsilon$ one concludes that $r\left(x_{\varepsilon}\right)>r_{0}$. In this case, $\Delta h>s / 2$, which contradicts the condition that $\Delta h<\varepsilon$.

\section{References}

[1] N. Nadirashvili, 'Hadamard's and Calabi-Yau's conjectures on negatively curved minimal surfaces', Invent. Math. 126 (1996), 457-465.

[2] P. Peterson, Riemannian Geometry (Springer, New York, 1998).

[3] S.-T. Yau, 'Harmonic functions on complete Riemannian manifolds', Comm. Pure Appl. Math. 28 (1975), 201-228.

ALBERT BORBÉLY, Faculty of Science, Department of Mathematics and Computer Science, Kuwait University, PO Box 5969, Safat 13060, Kuwait

e-mail: borbely.albert@gmail.com 